\title{
Clinical and Laboratory Outcomes of Angled Screw Channel Implant Prostheses: A Systematic Review
}

\author{
Vanya Rasaie ${ }^{1 \odot}$ Jaafar Abduo ${ }^{2}$ Mehran Falahchai ${ }^{3 \oplus}$ \\ ${ }^{1}$ Department of Prosthodontics, Dental Research Center, Dentistry \\ Research Institute, Tehran University of Medical Sciences, Tehran, Iran \\ 2 Department of Prosthodontics, Melbourne Dental School, \\ Melbourne University, Melbourne, Australia \\ ${ }^{3}$ Department of Prosthodontics, Dental Sciences Research Center, \\ School of Dentistry, Guilan University of Medical Sciences, Rasht, \\ Iran \\ Eur J Dent 2022;16:488-499.
}

\author{
Address for correspondence Vanya Rasaie, DDS, MSc, Dental \\ Research Center, Dentistry Research Institute, North Kargar \\ 1439955991, Tehran, Iran (e-mail: vanyarasaie@yahoo.com).
}

\begin{abstract}
Keywords

- angulated screw channel

- screw-retained prosthesis

- computer aided design- computer aided manufacturing

- implant
\end{abstract}

The purpose of this systematic review was to evaluate the clinical and laboratory outcomes of angled screw channel (ASC) restorations and to summarize the influencing factors. An electronic search of the English language literature was performed in four databases and enriched by manual searches. Retrieved studies were screened against the predefined exclusion and inclusion criteria. Eight clinical and seven laboratory studies were eligible for the analysis. The risk of bias for included observational studies was performed using the Newcastle-Ottawa quality assessment scale. Laboratory studies quality assessment method was adapted from previous published systematic reviews. Two clinical studies focused on technical outcomes and the rest reported the biological outcomes of the ASC restorations. Out of the seven laboratory studies, two studies investigated the fracture resistance of ASC restorations, four studies evaluated the reverse torque value of the nonaxially tightened screws, and one study evaluated both variables. The present review revealed that while the performance of ASC restorations is promising in short-term clinical studies, the evidence of their long-term reliability is still lacking. The laboratory studies indicated comparable fracture resistance results of the ASC restorations with the straight screw channel restorations. In addition, factors, such as initial torque value, configuration of the screw driver, screw design, abutment system, and the angulation of screw channel, were shown to influence the screw resistance to loosening.

\section{Introduction}

The implant-supported prosthesis is a successful strategy to restore dental esthetics and function. ${ }^{1}$ The definitive restoration can be attached to the implant by cement-retention or screw-retention mechanisms. ${ }^{2}$ While cementing implant

published online

February 21, 2022
DOI https://doi.org/ 10.1055/s-0041-1740298. ISSN $1305-7456$ restorations appears a simple procedure, clinical evidence suggests an association between biological complications and excess cement. ${ }^{3-6}$ Moreover, the difficulties in their maintenance and their retrievability have made screw-retention mechanism a more preferred choice. ${ }^{3,5,7}$ However, implants are not always placed in favorable positions for

\section{(C) 2022. The Author(s).}

This is an open access article published by Thieme under the terms of the Creative Commons Attribution License, permitting unrestricted use, distribution, and reproduction so long as the original work is properly cited. (https://creativecommons.org/licenses/by/4.0/)

Thieme Medical and Scientific Publishers Pvt. Ltd., A-12, 2nd Floor, Sector 2, Noida-201301 UP, India 
screw-retained restorations. This can be frequently encountered in the anterior maxilla due to centripetal resorption pattern and concave bone configuration. Further, angulated implant placement in the posterior regions can be indicated to avoid critical structures like maxillary sinus or mandibular canal. The treatment options to correct implant angulation may involve the use of an angled abutment with a cementretained restoration, or surgical bone augmentation to allow for more ideal implant placement. ${ }^{8}$ Alternatively, angle correction via intermediate abutments or restorations retained by a lateral screw could be considered. However, these systems have been shown to increase the treatment complexity, maintenance burden, and incur additional costs. ${ }^{8,9}$

Almost two decades ago, angled screw channel (ASC) abutment design (Dynamic Abutment; Talladium International Implantology) was introduced to allow restoring angulated implants with simple direct to fixture screwretained restoration. The ASC design uses a screw with a hexalobular head shape that can be engaged with a hexagonal faceted sphere screwdriver at various angles between 0 and 28 degrees with 360 -degree freedom of rotation. ${ }^{10}$ This allowed tightening the abutment screw at an orientation different from the center axis of the implant. Earlier, ASC abutments were casted on a hemisphere base via a burnout sleeve that could be rotated freely to direct the screw access channel away from the area of concern. ${ }^{10}$ Additionally, this concept was limited to certain implant systems. Recently, advancements in implant software programs and manufacturing systems have made it possible to design and fabricate ASC restorations digitally. Moreover, prefabricated titanium bases incorporating the ASC are available from some implant manufacturers. The versatility of ASC has been confirmed by a cone bean computed tomography (CBCT) analysis study that showed screw-retained restorations are achievable with the use of ASC abutments in 76\% of cases in the anterior maxilla. ${ }^{11}$ Despite the increased popularity, the efficiency and survival of the ASC systems remain unclear. The purpose of the present systematic review was to investigate laboratory and clinical outcomes of ASC restorations through the available literature to determine their survival and the influencing factors.

\section{Methods}

This systematic review adapted the Preferred Reporting Items for Systematic Reviews and Meta-Analysis (PRISMA) statement. The aim was to answer the following focused question "what are the outcomes of implants restored with angled screw channel prostheses?"

The main search strategy was developed for PubMed (-Table 1) and supplemented with additional search in Science Direct, Scopus, and Cochrane Library. The search was conducted in March 2021 and updated in May 2021. The search aimed to identify all the available clinical and laboratory studies on ASC. The inclusion criteria were peer-review publication, prospective or retrospective clinical study with at least 1 -year observation period after implant restoration, clinical study that evaluated biological and/or mechanical outcomes, clinical study that clearly listed outcome related to ASC restorations, and laboratory study that evaluated ASC performance variables with clinical relevance. The studies were excluded if they were not in English language, the ASCrelated data could not be determined, or the restorative stage details were not clearly stated. Duplicated articles from different searches were discarded by a reference manager software program (Endnote X9, Clarivate Analytics, Philadelphia, Pennsylvania, United States). Following this, the titles and abstracts were screened. The articles of interest were selected for full-text analysis and matching against the inclusion criteria. Further, the reference lists of the included studies were manually searched. Quality of the selected clinical studies was scored with Newcastle-Ottawa scale for nonrandomized studies ( - Table $\mathbf{2}$ ) which were designed to assess the quality of cohort studies based on selections of exposed and nonexposed cohorts, comparability influenced by the controls of risk factors, and completeness of outcomes. ${ }^{12}$ The risk of bias of the included laboratory studies

Table 1 Search strategy

\begin{tabular}{|c|c|}
\hline Search strategy & Query \\
\hline $\begin{array}{l}\text { Population: screw-retained } \\
\text { implant supported restorations }\end{array}$ & $\begin{array}{l}\text { (((Screw-retained[All Fields] AND implant[All Fields])) OR (“prostheses and } \\
\text { implants”[MeSH Terms] OR ("prostheses”[All Fields] AND "implants”[All Fields]) OR } \\
\text { "prostheses and implants"[All Fields])) OR (screw-retained[All Fields] AND restoration[All } \\
\text { Fields]) }\end{array}$ \\
\hline Intervention: ASC restorations & $\begin{array}{l}(((\text { abutment screw) AND (off[All Fields] AND “axis"[All Fields]))) OR ((“single”[All Fields] } \\
\text { AND implant[All Fields] AND restoration[All Fields]))) AND }(((((\text { angled[All Fields] AND } \\
\text { "screws"[All Fields] AND channel[All Fields])) OR (angulated[All Fields] AND screws”[All } \\
\text { Fields])) OR (non-axial[All Fields] AND "screws"[All Fields] AND channel[All Fields])) OR } \\
\text { (Abutment[All Fields] AND "screws"[All Fields] AND channel[All Fields])) OR (two[All Fields] } \\
\text { AND piece[All Fields] AND abutment[All Fields])) }\end{array}$ \\
\hline Outcome: & $\begin{array}{l}(((((\text { reverse[All Fields] AND (“torque”[MeSH Terms] OR “torque”[All Fields]))) OR (technical } \\
{[\text { All Fields] AND (“complications”[Subheading] OR “complications”[All Fields]))) OR }} \\
\text { (mechanical[All Fields] AND complication[All Fields])) OR (fractures[All Fields] AND } \\
\text { resistance[All Fields])) OR "survival rate”[MeSH Terms] }\end{array}$ \\
\hline
\end{tabular}


Table 2 Quality assessment of selected clinical studies using Newcastle-Ottawa scale for cohort studies

\begin{tabular}{|c|c|c|c|c|c|c|c|c|c|}
\hline $\begin{array}{l}\text { Quality } \\
\text { assessment } \\
\text { criteria }\end{array}$ & Acceptable & $\begin{array}{l}\text { Greer } \\
\text { et } \mathrm{al}^{23}\end{array}$ & $\begin{array}{l}\text { Anitua } \\
\text { et } \mathrm{al}^{25}\end{array}$ & $\begin{array}{l}\text { Tallarico } \\
\text { et } \mathrm{al}^{27}\end{array}$ & $\begin{array}{l}\text { Friberg and } \\
\text { Ahmadzai }^{24}\end{array}$ & $\begin{array}{l}\text { Pol } \\
\text { et } \mathrm{al}^{22}\end{array}$ & $\begin{array}{l}\text { Anitua } \\
\text { et } \mathrm{al}^{26}\end{array}$ & $\begin{array}{l}\text { Shi } \\
\text { et } \mathrm{al}^{28}\end{array}$ & $\begin{array}{l}\text { Nastri } \\
\text { et } \text { al }^{29}\end{array}$ \\
\hline $\begin{array}{l}\text { Representativeness } \\
\text { of exposed cohort? }\end{array}$ & $\begin{array}{l}\text { Representative of } \\
\text { average adult in } \\
\text { community }\end{array}$ & 1 & & & 1 & & & & \\
\hline $\begin{array}{l}\text { Selection of } \\
\text { nonexposed cohort }\end{array}$ & $\begin{array}{l}\text { Drawn from same } \\
\text { community as } \\
\text { exposed cohort }\end{array}$ & & & & & & & & \\
\hline $\begin{array}{l}\text { Ascertainment of } \\
\text { exposure }\end{array}$ & Secured records & 1 & 1 & 1 & 1 & 1 & 1 & 1 & 1 \\
\hline $\begin{array}{l}\text { Demonstration } \\
\text { that outcome of } \\
\text { interest not present } \\
\text { at start of the study }\end{array}$ & Yes & 1 & 1 & 1 & 1 & 1 & 1 & 1 & 1 \\
\hline $\begin{array}{l}\text { Study controls for } \\
\text { the degree of screw } \\
\text { access angulation }\end{array}$ & Yes & & 1 & & & & 1 & 1 & \\
\hline $\begin{array}{l}\text { Study controls for } \\
\text { additional risk } \\
\text { factor? }\end{array}$ & restorative material & 1 & 1 & 1 & & 1 & 1 & 1 & \\
\hline $\begin{array}{l}\text { Assessment of } \\
\text { outcome }\end{array}$ & Secure records & 1 & 1 & 1 & 1 & 1 & 1 & 1 & 1 \\
\hline $\begin{array}{l}\text { Follow-up long } \\
\text { enough }\end{array}$ & Follow-up ( $>1$ year) & & & 1 & & & 1 & & 1 \\
\hline $\begin{array}{l}\text { Adequacy of follow- } \\
\text { up }\end{array}$ & $\begin{array}{l}\text { Small number of } \\
\text { subject loss }\end{array}$ & 1 & 1 & 1 & & 1 & 1 & 1 & 1 \\
\hline \multicolumn{2}{|c|}{$\begin{array}{l}\text { Overall quality score (maximum }=9 \text { ) }>7 \text { : } \\
\text { good } / 5-7 \text { : fair } /<5 \text { poor }\end{array}$} & 6 & 6 & 6 & 4 & 5 & 7 & 6 & 5 \\
\hline
\end{tabular}

were assessed by using an adaptation of the methods applied in two previous systematic reviews. ${ }^{13,14}$ Descriptions of the following parameters were used to assess each article's risk of bias ( - Table 3): sample size calculation, presence of a control group, type of component used (genuine/nongenuine), statistical analysis performed, reliable analytical methods or statistical indicators, blinding of the evaluation assessors, and utilizing clinically relevant restoration material. A "yes" was assigned where the parameter was reported in the text and a "no" if the information was absent. The risk of bias was classified according to the sum of "yes" received as follows: 1 to $3=$ high, 4 to $5=$ medium, and 6 to $7=$ low risk of bias.

\section{Results}

The initial electronic search yielded 358 publications. After elimination of duplicates, 326 remained for title and abstract review. Twenty-seven articles were selected for full-text analysis, 13 of which were laboratory and clinical studies that fulfilled the inclusion criteria. Fourteen studies were excluded as listed in - Table 4 along with the reasons of exclusion. The supplementary manual search through the bibliography of the included studies yielded two additional articles. Therefore, 15 publications were eligible for the current review (-Fig. 1). The included studies were published between 2017 and 2021, with the majority being published in the past 2 years. Out of seven laboratory investigations, two studies evaluated the fracture strength of the zirconia crowns with ASC ( - Table 5), ${ }^{15,16}$ four studies evaluated the reverse torque value of nonaxially tightened screws (-Table 6), ${ }^{17-20}$ and one examined both variables. ${ }^{21}$ Only four studies reported the exact direction of force in their investigations that was 30 degrees for all of them. ${ }^{15,17,19,21} \mathrm{~A}$ total of eight nonrandomized cohort studies (four prospective and four retrospective) reported the outcomes of 281 implants restored with ASC restorations in 254 patients $\left(-\right.$ Table 7). ${ }^{22-28}$ Of these, two investigations focused on technical complications ${ }^{23,25}$ and the rest reported the technical and biological outcomes of ASC restorations. ${ }^{22,24,26-28}$ Six studies included only single-crown restorations $^{22-24,27,28}$ and two studies included partial or full arch restorations. ${ }^{25,26}$ All but two studies reported cumulative survival rates after at least 1 year of loading.

Drew et $a l^{16}$ and Garcia-Hammaker et $\mathrm{al}^{15}$ examined the fracture strength of the two piece CAD/CAM monolithic zirconia crown with 25-degree ACS by using cyclic and static loads, respectively. Drew et al ${ }^{16}$ showed no statistically significant difference in the mean number of cyclic loads to failure between ASC and straight screw channel (SSC) 
Table 3 Quality assessment and risk of bias of laboratory studies considering aspects (reported in "Materials and Methods" section)

\begin{tabular}{|c|c|c|c|c|c|c|c|c|}
\hline Author (year) & $\begin{array}{l}\text { Sample size } \\
\text { calculation }\end{array}$ & $\begin{array}{l}\text { Control } \\
\text { group }\end{array}$ & $\begin{array}{l}\text { Genuine } \\
\text { component } \\
\text { used }\end{array}$ & $\begin{array}{l}\text { statistical } \\
\text { analysis } \\
\text { performed }\end{array}$ & $\begin{array}{l}\text { reliable } \\
\text { analytical } \\
\text { methods or } \\
\text { statistical } \\
\text { indicators }\end{array}$ & $\begin{array}{l}\text { blinding } \\
\text { of the } \\
\text { evaluation } \\
\text { assessors }\end{array}$ & $\begin{array}{l}\text { Utilization of } \\
\text { clinically } \\
\text { relevant } \\
\text { restoration } \\
\text { material }\end{array}$ & $\begin{array}{l}\text { Risk } \\
\text { of bias }\end{array}$ \\
\hline Goldberg et al $(2019)^{21}$ & No & Yes & No & Yes & Yes & No & No & High \\
\hline Hu et al $(2019)^{20}$ & Yes & Yes & Yes & Yes & Yes & No & $\mathrm{NA}^{\circ}$ & Medium \\
\hline Opler et al (2019) ${ }^{18}$ & No & Yes & No & Yes & Yes & No & NA & High \\
\hline Swamidass et al (2021) ${ }^{17}$ & No & Yes & Yes & Yes & Yes & No & Yes & Medium \\
\hline Drew et al $(2020)^{16}$ & No & Yes & Yes & Yes & Yes & No & Yes & Medium \\
\hline Garcia-Hammaker et al $(2021)^{15}$ & Yes & Yes & Yes & Yes & Yes & No & Yes & Low \\
\hline Mulla et al $(2021)^{19}$ & No & Yes & Yes & Yes & Yes & No & Yes & Medium \\
\hline
\end{tabular}

Abbreviation: NA, not applicable.

Table 4 Reasons for exclusion of discarded studies after full-text analysis

\begin{tabular}{|c|c|c|c|}
\hline Reasons for exclusion & $\begin{array}{l}\text { Did not evaluated ASC } \\
\text { restorations }\end{array}$ & $\begin{array}{l}\text { Not indicative of variables } \\
\text { with clinical relevance }\end{array}$ & $\begin{array}{l}\text { No data about } \\
\text { prosthetic stage of treatment }\end{array}$ \\
\hline Excluded studies & $\begin{array}{l}\text { Anitua et al }\left.\right|^{40} \\
\text { Chen and Pan } \\
\text { Guljé et al }^{42} \\
\text { Hotinski and Dudley } \\
\text { Lin et al }{ }^{30} \\
\text { Menéndez-Collar et al }\left.\right|^{44} \\
\text { Mokhtarpour et al }{ }^{45} \\
\text { Paolantoni et al }{ }^{46} \\
\text { Vélez et al }\end{array}$ & $\begin{array}{l}\text { Edmondson et al }{ }^{11} \\
\text { González-Martín and Veltri } 48 \\
\text { Farré-Berga et al }{ }^{49} \\
\text { Farronato et al }{ }^{50}\end{array}$ & Wang et $\mathrm{al}^{51}$ \\
\hline
\end{tabular}

Abbreviation: ASC, angled screw channel.

crowns. However, the SSC crowns survived a greater number of cycles prior to failure. Garcia-Hammaker et $\mathrm{al}^{15}$ reported a significantly higher mean fracture load by 2.4 times and maximum load before failure by 1.8 times for the SSC crowns. Despite the superior fatigue loading of SSC, the specimens in both groups resisted the physiologic loads and failure occurred at loads that resembled a parafunctional situation. Goldberg et $\mathrm{al}^{21}$ used external connection hexagon implants for the comparison of the fracture strength of SSC gold screw abutments with 0,20, and 28 degrees of Dynamic abutments casted to nickel-chromium ( $\mathrm{Ni}-\mathrm{Cr}$ ) crowns, after aging in a mastication simulator. The study reported screw fracture before implants' mechanical failure in $17.8 \%$ of specimens with only $0.03 \%$ of fractures attributed to the ASC restorations. Furthermore, no significant difference was reported among the fracture strength values of the studied groups.

In the same study, Goldberg et $\mathrm{al}^{21}$ compared the reverse torque value (RTV) of the nonaxially tightened dynamic abutment screws (DAS) with 0-degree DAS and SSC gold screw. Although not statistically significant, the 0-degree DAS demonstrated the highest RTVs, while the 28-degree DAS showed the lowest values. The study suggested that increased off-axis loading resulted in higher tensile forces on abutment screws.
Hu et $\mathrm{al}^{20}$ showed that the screwdriver insertion angulation has a significant impact on the RTVs of the abutment screws tightened in 0-, 10-, and 20-degree angles. The lowest mean RTV was in the 20-degree group and was described as the loss of applied torque due to increased screwdriver angle from the action of force. The highest mean RTV was shown in 10-degree specimens. This was explained by the possibility of a more intimate engagement of the screwdriver tip and the abutment screw at 10-degree angulation for the chosen system. Another study investigated the effect of screwdriver insertion angulation on the RTVs, input torque values delivered at various angulations (0, 10, 15, 25, and 28 degrees), and the transmitted output torque values in five different angulations. While the study revealed no significant difference in input torque values among the groups, the mean RTV between the 0 - and 28-degree groups was significantly different with lower values for the 28-degree group. Regarding the mean output torque values, which was measured by the strain induced in the screw body, the results revealed no significant difference between 0 - and 15-degree angulations. However, significantly lower output torque transmission to abutment screws was found when the angulation increased to 25 and 28 degrees. Furthermore, photography of the specimens revealed evidence of wear at the screw head 


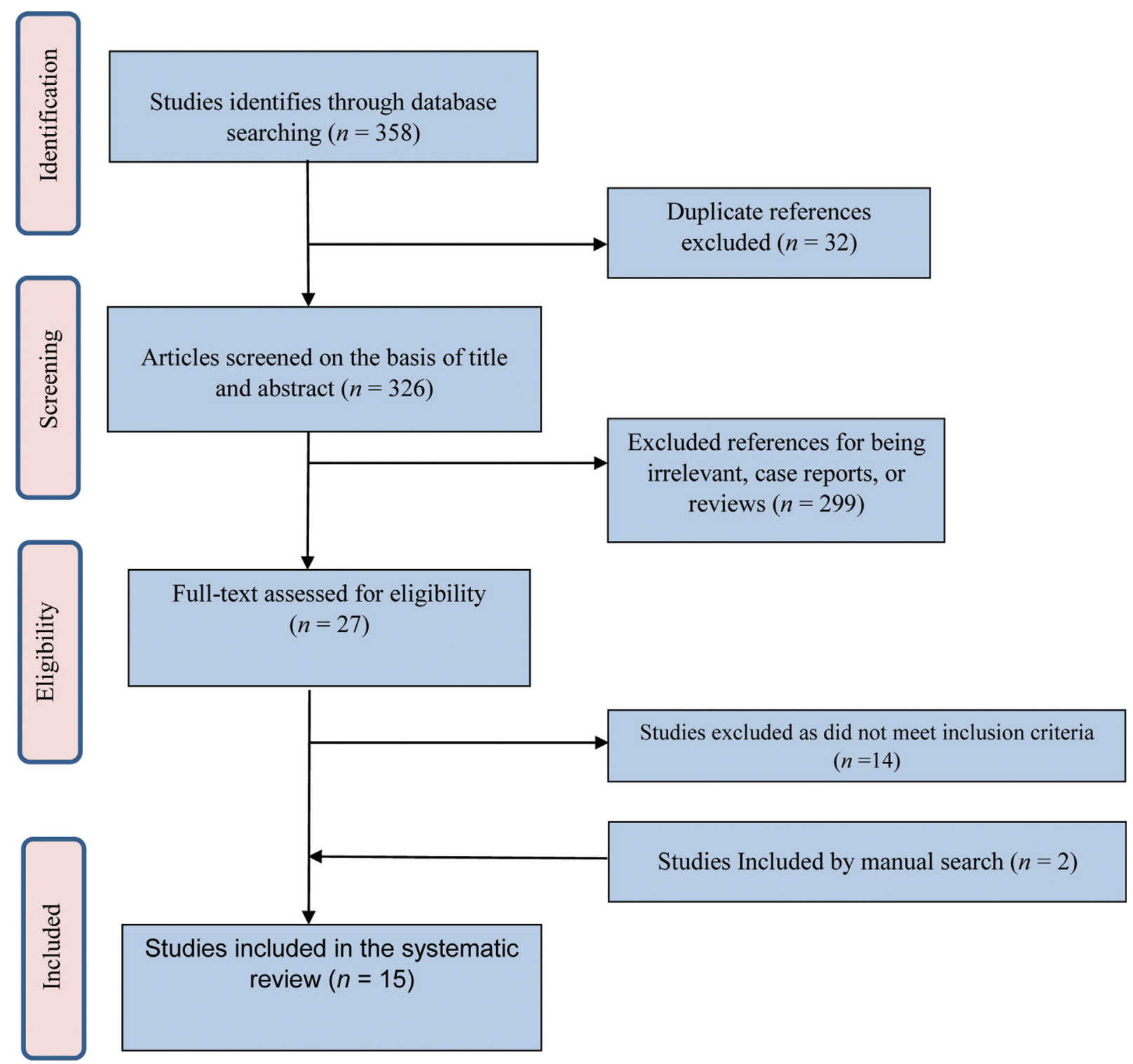

Fig. 1 Flowchart for search process according to PRISMA guideline. PRISMA, preferred reporting items for systematic reviews and metaanalyses.

predominantly with the 28-degree group, suggesting slipping of the driver. ${ }^{18}$

Swamidass et al ${ }^{17}$ investigated the differences in RTVs of ASC abutments from different manufacturers before and after simulated aging by temperature and cyclic loading. The RTV was measured 10 minutes after tightening as the initial value and subsequent to aging as the ultimate value. The percentage of the differences of RTVs was calculated and analyzed. In general, while the systems with higher initial torque value showed lower percentage torque loss, the differences between the 0 - and 20-degree groups were not significant. On the other hand, when the abutment screws and implant were from the same manufacturer, no significant difference between the SSC and ASC groups was identified. In contrast, differences between the SSC group with genuine abutment screws and the ASC group with abutment screws from alternate brands were significant. An additional finding was the wear of the screw head against the zirconia crown in groups with friction fitted two-piece zirconia abutments (Nobel Biocare). Congruent with this study, Mulla et $\mathrm{al}^{19}$ evaluated a higher magnitude of cyclic forces for a longer duration of time on 25-degree access channel restorations from three different manufacturers. RTVs were measured 24 hours after initial tightening and after simulated 5 years of functional load. Additionally, their study analyzed the deviation of the input torque value from the target value recommended by the manufacturer. Two hexalobular systems (Nobel Biocare and Dess Dental Smart Solutions) delivered significantly lower input torque values at 25 degrees compared with the SSC group. Nonetheless, their measured RTVs were not significantly different from the RTVs of SSC group. The 25-degree dynamic Ti-base system revealed insignificant input value torque deviation compared with 0-degree group. Unlike the other three study groups, this hexalobular system exhibited a high amount of torque loss at both times of RTV measurement. The statistical 


\begin{tabular}{|c|c|c|c|}
\hline 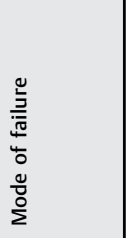 & 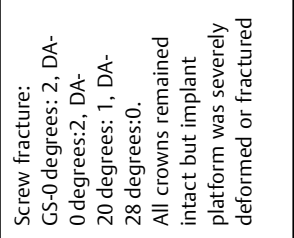 & 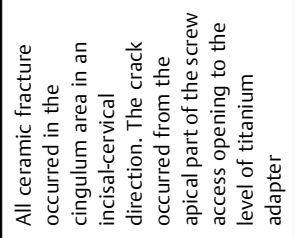 & 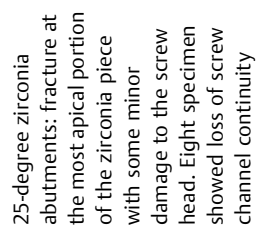 \\
\hline $\begin{array}{l}\frac{\underline{y}}{\bar{z}} \\
\stackrel{a}{x}\end{array}$ & 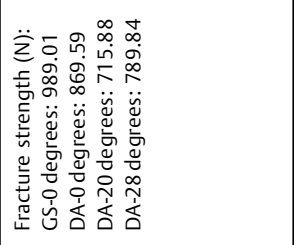 & 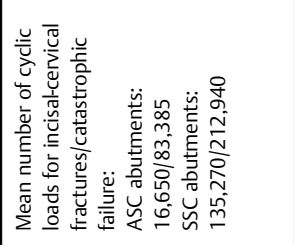 & 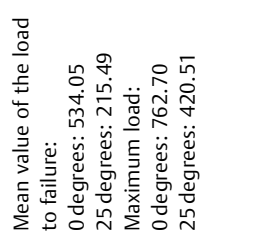 \\
\hline 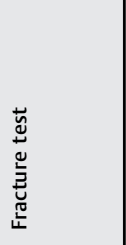 & 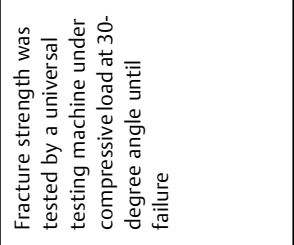 & 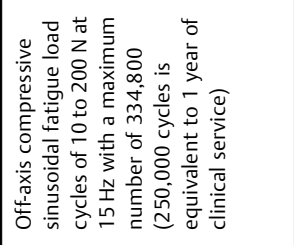 & 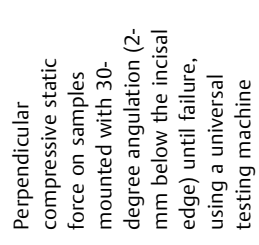 \\
\hline 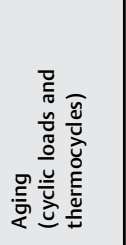 & 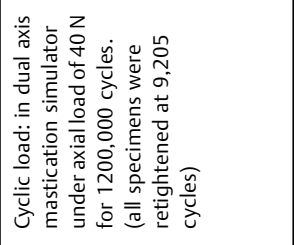 & & 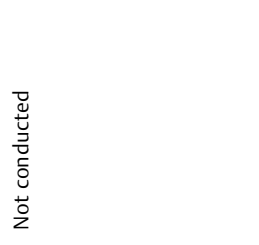 \\
\hline 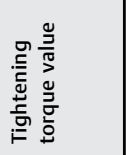 & 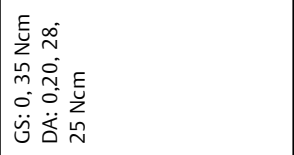 & 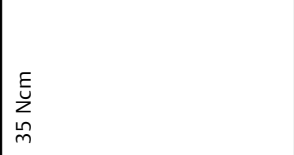 & $\underset{\substack{E \\
m}}{E}$ \\
\hline 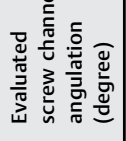 & 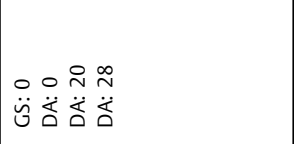 & $0 \stackrel{n}{N}$ & $0 \stackrel{\Perp n}{\sim}$ \\
\hline 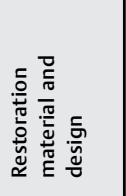 & 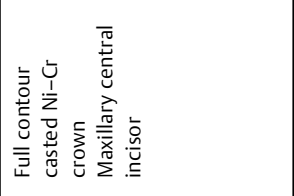 & 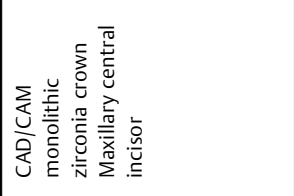 & 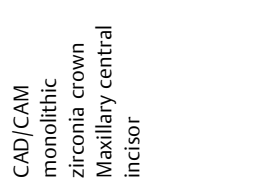 \\
\hline 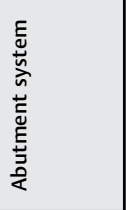 & 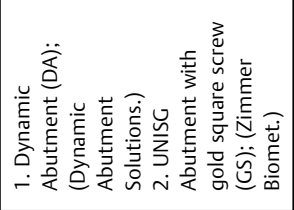 & 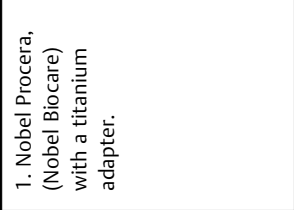 & 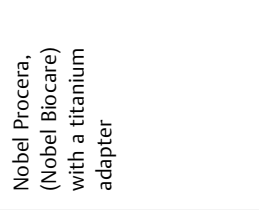 \\
\hline 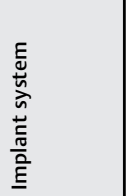 & 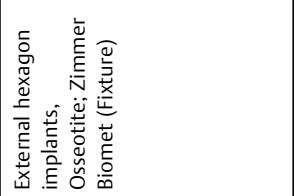 & 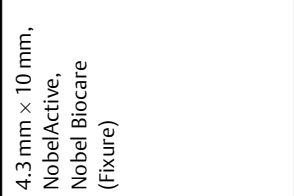 & 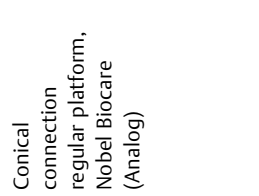 \\
\hline 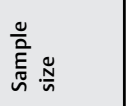 & $\stackrel{\pi}{\pi}$ & $\begin{array}{l}\| \\
\| \\
=\end{array}$ & $\stackrel{\circ}{I I}$ \\
\hline 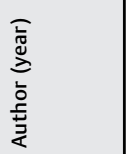 & 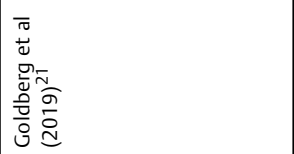 & 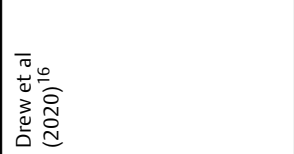 & 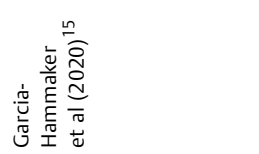 \\
\hline
\end{tabular}




\begin{tabular}{|c|c|c|c|c|c|}
\hline 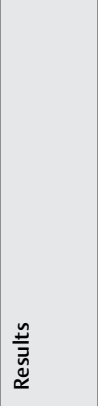 & 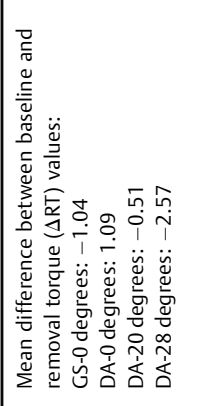 & 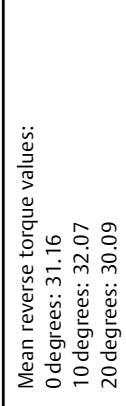 & 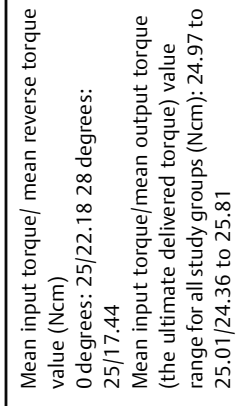 & 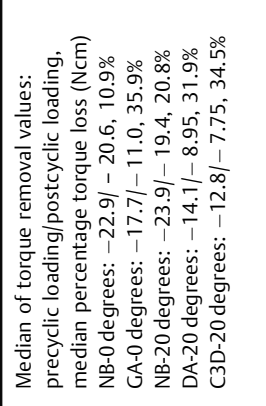 & 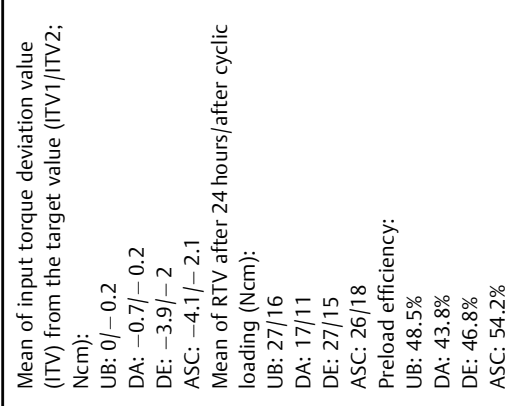 \\
\hline 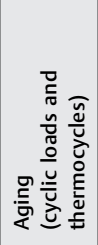 & 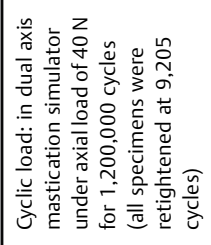 & 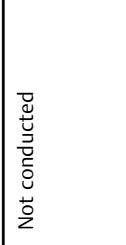 & 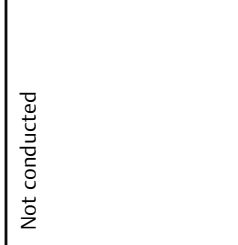 & 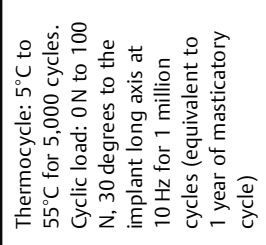 & 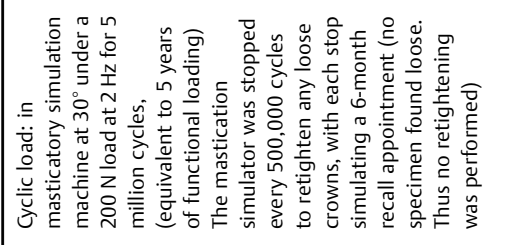 \\
\hline 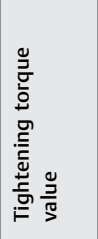 & 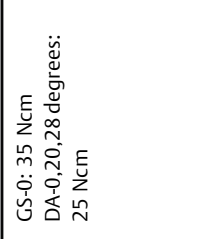 & 㣽 & 昼 & 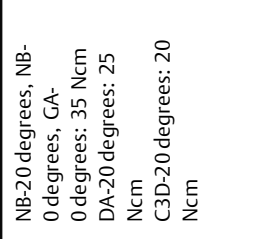 & 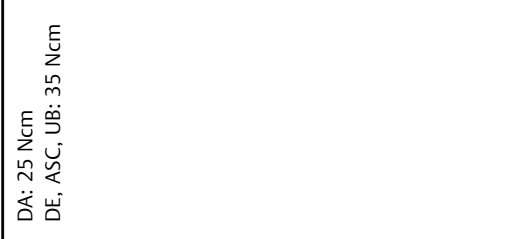 \\
\hline 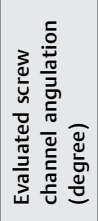 & 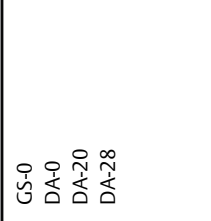 & 유유 & 으는 $\stackrel{\infty}{\sim} \stackrel{\infty}{\sim}$ & 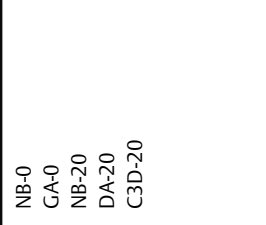 & 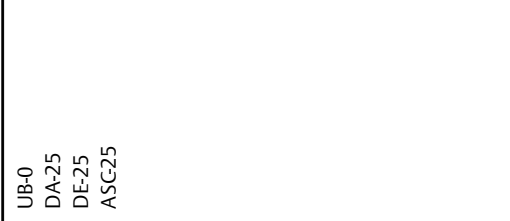 \\
\hline 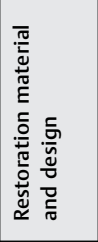 & 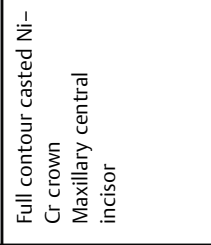 & 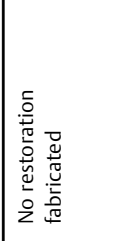 & 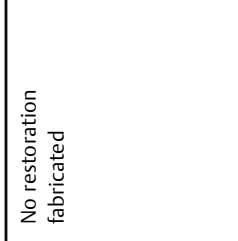 & 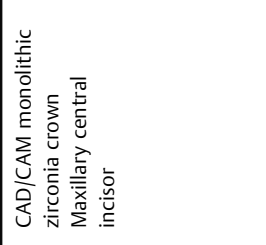 & 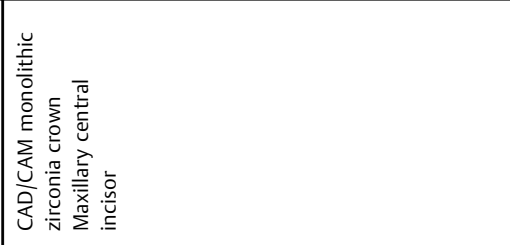 \\
\hline 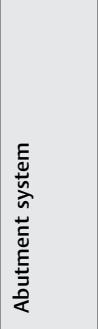 & 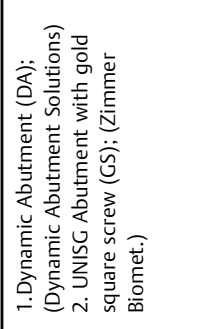 & 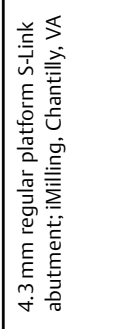 & 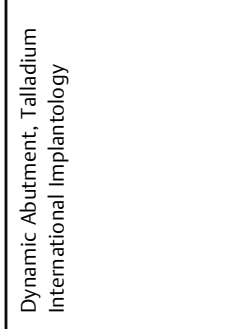 & 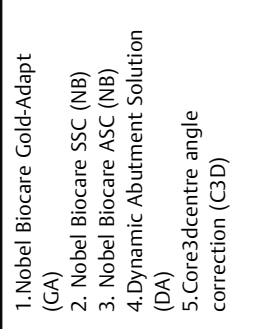 & 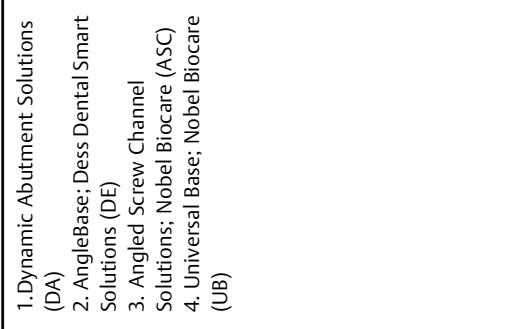 \\
\hline 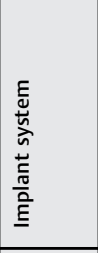 & 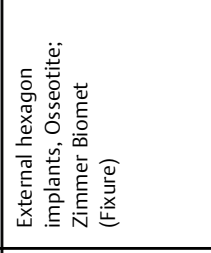 & 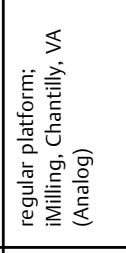 & 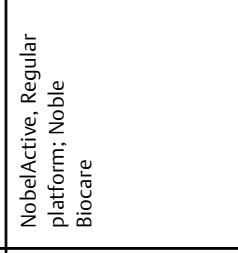 & 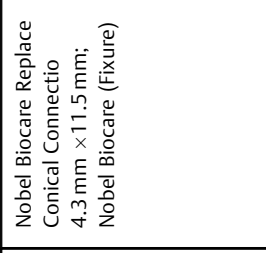 & 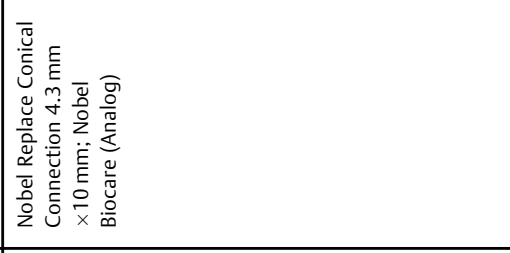 \\
\hline 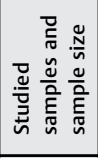 & $\begin{array}{l}\pi \\
\| \\
=\end{array}$ & $\begin{array}{l}0 \\
I I \\
=\end{array}$ & $\begin{array}{l}\circ \\
\text { III } \\
=\end{array}$ & $\begin{array}{l}\circ \\
I \\
=\end{array}$ & 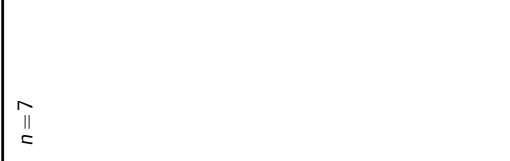 \\
\hline 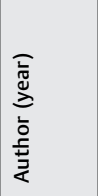 & 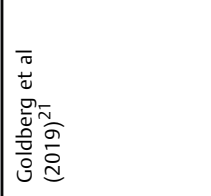 & 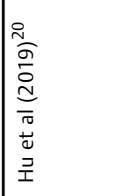 & 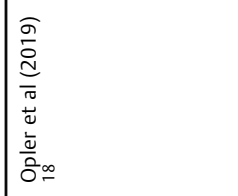 & 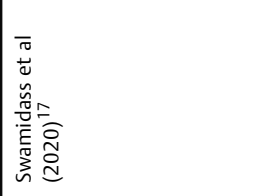 & 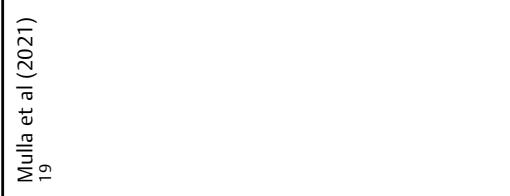 \\
\hline
\end{tabular}




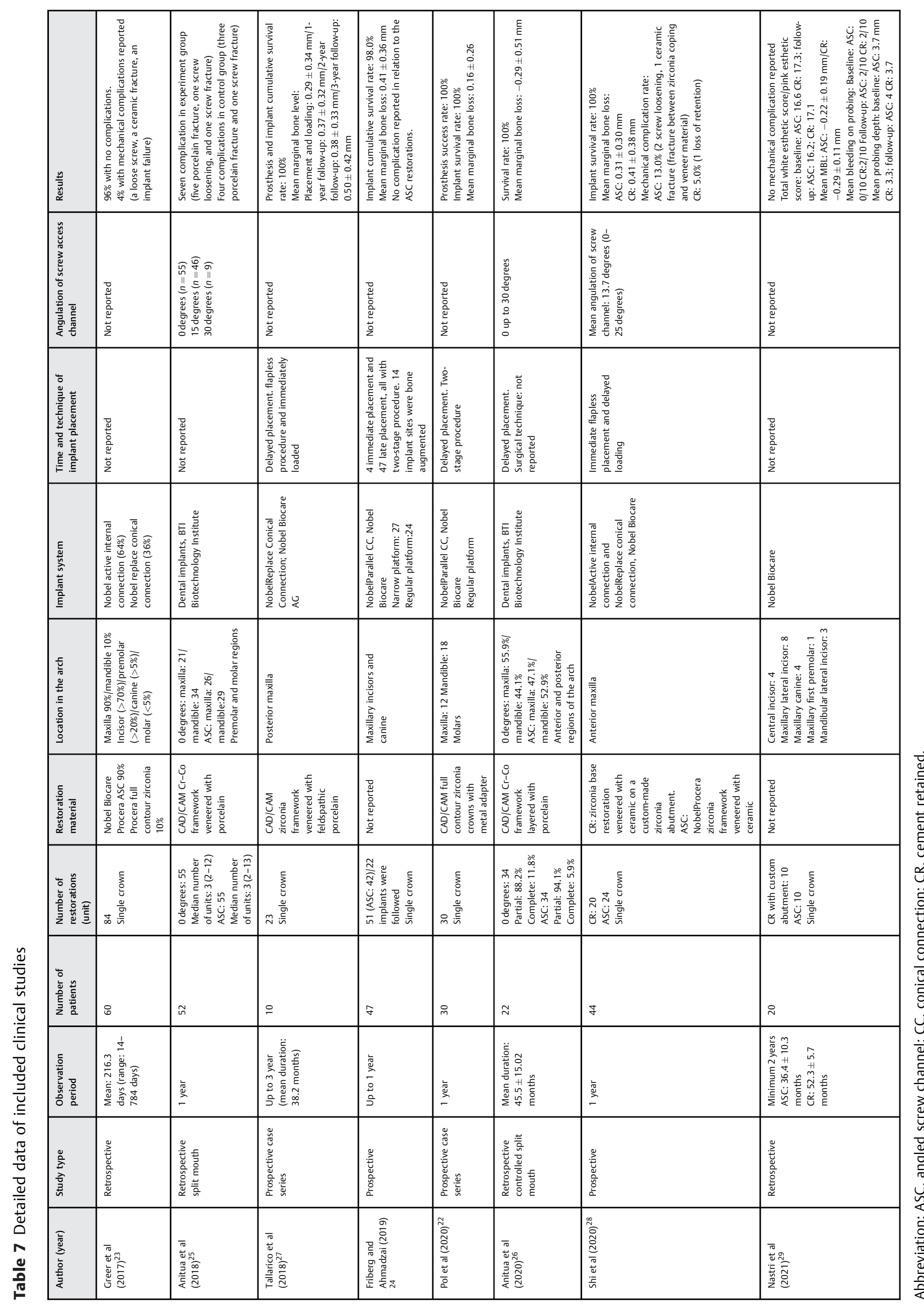

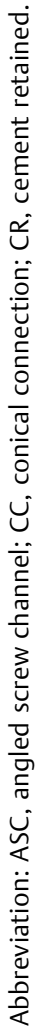


analysis of this study revealed significant differences in mean RTVs for both 24-hour RTV and after cyclic loading among all groups. In addition, the RTV means before and after cyclic loading for each group were significantly different. Furthermore, out of five catastrophic failures reported with the 25degree groups, three were related to zirconia fracture initiated from the area surrounding Ti-base. Other two failures were Ti-base and screw head fracture in systems with cemented two-piece zirconia abutment. Overall, no significant difference was found in the survival rate among the groups.

Overall outcomes from the seven clinical studies showed a high survival rate for both the ASC restorations (88-100\%) and the dental implants (98-100\%) with low mean marginal bone loss (MBL; 0.16-0.41 mm). However, the majority of the studies were conducted for a short duration of time (1 year). Ceramic fracture was the most frequent complication that was reported in three studies (seven events), ${ }^{23,25,28}$ mostly related to 15-degree metal-ceramic multiunit restorations with equal incidents in both arches (two in mandible and two in maxilla). Poor occlusal management was the attributed causes of ceramic fracture in the Greer et al study. ${ }^{23}$ Screw loosening was reported in three studies (four events), ${ }^{23,25,28}$ and screw fracture was reported in one study (one event). ${ }^{25}$

Two studies included data for ASC and SSC restorations. ${ }^{25,26}$ These studies evaluated multiunit metal ceramic fixed prosthesis in the posterior region (premolar and molar) of both arches, whereas the abutment screws were tightened either in axial or nonaxial direction. During follow-up, a total of 11 technical events were reported, 7 in the ASC group and 4 in SSC group (-Table 6). However, analysis of the results indicated that neither the frequency of technical complications nor the implants' survival and MBL were affected by the angulation of the screw channel. Tallarico et $\mathrm{al}^{27}$ used ASC zirconia abutments for immediate restoration of tilted implants placed in the posterior region of the atrophic maxilla. Although the studied sample size was limited, no biological or technical complications were reported in the 3year follow-up. The authors suggested that the combination of tilted implants and ASC abutments might be a safe alternative to maxillary sinus floor augmentation procedures when patients refuse additional surgical procedures. Friberg and Ahmadzai ${ }^{24}$ and Pol et al $^{22}$ evaluated the ASC restorations with conical connection implants for replacement of single missing teeth in incisor/canine and molar region, respectively. None of the studies indicated any complication after 1 year of function. Shi et $\mathrm{al}^{28}$ compared the technical and biological outcomes of ASC and cement-retained single restoration in anterior maxilla after 1 year of loading. Their report indicated that while the difference in MBL was insignificant among the two study groups, bleeding on probing percentage was significantly higher in the cementretained group. In addition, four events of technical complications were reported. Screw loosening and ceramic chipping were the complications associated with the ASC cohorts. Contrary to these findings, Nastri et $\mathrm{al}^{29}$ reported no mechanical complication and no significant difference in bleeding on probing among their study cohorts (cement- retained versus ASC single crowns) through the 2-year follow-up. Additionally, the differences in probing depth, mean MBL, and white esthetic score between the two study groups were insignificant. However, the cement-retained restorations had significantly higher pink esthetic scores both in baseline and follow-up recordings.

\section{Discussion}

This systematic review critically appraised the existing evidence from the laboratory and the clinical studies on the implant-supported ASC restorations. Concerning laboratory studies, fracture strength and screw resistance to loosening were investigated. According to fracture strength studies, the ASC restorations appear to fail at less cycles of loads and lower forces than SSC. However, since they sustained the expected physiological forces, the ASC restorations were considered mechanically comparable with SSC counterparts. ${ }^{15,16,21}$ Fractographic examinations of anterior twopiece zirconia abutments revealed that critical cracks were initiated in the cingulum from the most apical part of the screw access channel in the friction-fitted system. ${ }^{15,16,19}$ Consistent with this finding, some clinical studies have reported early catastrophic failures with the same pattern in two-piece friction fitted zirconia abutments in anterior and premolar SSC restorations. ${ }^{30,31}$ As the screw head seats on the internal surface of the zirconia restoration, hoop stresses and/or incompatible hardness between the zirconia and titanium components may be responsible for such pattern of failure. ${ }^{16,17,19}$ This could suggest that the abutment-titanium base interface design might play more considerable role than the ASC in the long-term performance of the hexalobular systems. On the other hand, as the angulation of the screw channel is increased, the bulk of the palatal walls of anterior zirconia abutments is reduced. ${ }^{15,16}$ This could lead to a weak point in zirconia restorations where the thickness my reach to less than $0.7 \mathrm{~mm} .{ }^{32}$ Thus, precaution should be taken when using ASC until more robust evidence is available on the interaction between the angulation amount and abutment thickness. Since ASC restorations were mostly required in the anterior maxillary region, ${ }^{11}$ the investigated restoration materials by laboratory studies (monolithic zirconia or full-contour $\mathrm{Ni}-\mathrm{Cr}$ crowns) may not be fully representative of the clinical application of ASC in highly demanding esthetic clinical situation. Therefore, further studies are required to determine the reliability of other restorative materials with ASC.

When resistance to screw loosening is considered, studies showed that the RTV was influenced by initial torque value, ${ }^{17,19}$ configuration of the screwdriver, ${ }^{20}$ screw design, ${ }^{17,19}$ abutment system, ${ }^{17,19}$ and angulation of the screw channel. ${ }^{17-21}$ As the torque is applied to an abutment screw, it elongates and the threaded surface elastically deforms. Adequate screw elastic elongation, referred to as preload, could secure the implant-abutment joint by appropriate clamping forces. ${ }^{33} \mathrm{~A}$ reduction in the input torque could compromise the screw joint by reducing the applied preload. ${ }^{34} \mathrm{~A}$ consistent outcome of the two studies 
investigated various ASC systems indicated that the higher the initial torque value, the less susceptible the screw to loosening. ${ }^{17,19}$ Although the measurement methods were different, two studies showed that the actual torque delivered to the screw is reduced when the insertion angle of the screwdriver exceeds 15 degrees (25 and 28 degrees). ${ }^{18,19}$ However, RTVs of screws from different systems showed variable behaviors. For example, while 25-degree Nobel Biocare and 25-degree Dess abutment screws performed similarly, ${ }^{19}$ the 28 -degree DAS exhibited a significant $23 \%$ reduction in RTV when compared with the 0 -degree control group. ${ }^{18}$

Intimate engagement of the screwdriver and screw head showed to play a role in the amount of the delivered torque. This is further influenced by the screwdriver's sphere and facet design, as well as, the surface treatment of the screw head. ${ }^{17-20}$ Likewise, difficulty in the engagement of the screwdriver, which was reported with certain systems, led to stripping and wear of the screw head that might impact the crown retrievability and the usability of the screws after multiple tightening in long duration of time. ${ }^{17,18}$

The use of nongenuine component can influence the RTV and may jeopardize the joint stability of the implant-abutment complex. ${ }^{17} \mathrm{~A}$ proper match and integration of the components within an implant system is important, especially when using implants with conical/internal connection as shown by the included studies. ${ }^{35}$ Further investigations is required to evaluate the RTV with other types of implant connection.

The majority of the included clinical studies reported no major complications after 1 year of treatment of posterior regions with conical connection Nobel Biocare implants and ASC restorations. ${ }^{22,25-27}$ Consistent with these, the anterior ASC restorations revealed favorable clinical performance as well. $^{23,24,29}$ Technical complications were reported in three studies ${ }^{23,25,28}$ with no significant differences between ASC and SSC or cement-retained restorations. ${ }^{25,26,28} \mathrm{~A}$ previous clinical study with up to 9 years of follow-up has reported that two-piece zirconia SSC abutments with bonded titanium insert can be a suitable option for anterior and premolar region. However, in the molar area, the use of the same abutment without a complete metal-to-metal connection platform (friction-fitted titanium insert) to support the restorations have led to a high incidence of fracture. ${ }^{30}$

The data on MBL around the ASC restorations revealed favorable outcomes. Previous systematic reviews have indicated that changes in the crestal bone level is not significantly affected by angulated implant placement as compared with axial placement. ${ }^{36,37}$ However, other factors related to the abutment design may affect the amount of MBL, such as the abutment height, and contour, ${ }^{38}$ and repeated disconnection and reconnection of the abutment. ${ }^{39}$ In the present systematic review, this complementary information was mostly missing. In addition, although the MBL in ASC and SSC were comparable, the number of studies comparing them was scares.

While ASC restorations show promising short-term results, some clinical questions are yet to be answered. For example, there is a need to determine their long-term clinical performance, cost-effectiveness, and the management of their complications. Thus, the availability of ASC should not be a justification for injudicious angular implant placement.

\section{Limitations}

The present systematic review is limited with the fact that few clinical studies provided clear comparison between ASC and SSC. In addition, the fair quality of the majority of clinical studies and high-to-medium risk of bias of the laboratory mandate the need for stronger future evidences. Another limitation is that the clinical data were incomplete regarding the amount of angle correction. Moreover, most of the clinical studies had only 1-year duration of observation which does not reflect the long-term performance of ASC. Thus, the current evidence for use of ASC is limited and a more robust clinical guidance on the application of ASC abutments is needed.

\section{Conclusion}

Within the limitation of this systematic review, the following inferences can be drawn:

1. According to laboratory studies, the fracture resistance of SSC and ASC restorations were comparable. The incidence of screw loosening might be lower in ASC systems with higher insertion torque and genuine components.

2. According to clinical studies, although the ASC restorations demonstrated favorable performance in anterior and posterior regions of the mouth in short-term, evidence is insufficient to determine their long-term survival.

\section{Funding \\ None.}

Conflict of Interest

None declared.

\section{References}

1 Moraschini V, Poubel LA, Ferreira VF, Barboza EdosS. Evaluation of survival and success rates of dental implants reported in longitudinal studies with a follow-up period of at least 10 years: a systematic review. Int J Oral Maxillofac Surg 2015;44(03): 377-388

2 Dario LJ. Implant angulation and position and screw or cement retention: clinical guidelines. Implant Dent 1996;5(02):101-104

3 Sailer I, Mühlemann S, Zwahlen M, Hämmerle CH, Schneider D. Cemented and screw-retained implant reconstructions: a systematic review of the survival and complication rates. Clin Oral Implants Res 2012;23(Suppl 6):163-201

4 Wittneben JG, Millen C, Brägger U. Clinical performance of screwversus cement-retained fixed implant-supported reconstructions-a systematic review. Int J Oral Maxillofac Implants 2014; 29(suppl):84-98

5 Michalakis KX, Hirayama H, Garefis PD. Cement-retained versus screw-retained implant restorations: a critical review. Int J Oral Maxillofac Implants 2003;18(05):719-728 
6 Albrektsson T, Canullo L, Cochran D, De Bruyn H. "Peri-implantitis": a complication of a foreign body or a man-made "disease". Facts and fiction. Clin Implant Dent Relat Res 2016;18(04): 840-849

7 Millen C, Brägger U, Wittneben JG. Influence of prosthesis type and retention mechanism on complications with fixed implantsupported prostheses: a systematic review applying multivariate analyses. Int J Oral Maxillofac Implants 2015;30(01):110-124

8 Cavallaro J Jr, Greenstein G. Angled implant abutments: a practical application of available knowledge. J Am Dent Assoc 2011;142 (02):150-158

9 Sethi A, Sochor P. The lateral fixation screw in implant dentistry. Eur J Prosthodont Restor Dent 2000;8(01):39-43

10 Berroeta E, Zabalegui I, Donovan T, Chee W. Dynamic abutment: a method of redirecting screw access for implant-supported restorations: technical details and a clinical report. J Prosthet Dent 2015;113(06):516-519

11 Edmondson EK, Trejo PM, Soldatos N, Weltman RL. The ability to screw-retain single implant-supported restorations in the anterior maxilla: a CBCT analysis. J Prosthet Dent 2021;S0022-3913 (21):00032-00039

12 Wells GSB, O'Connell D, Peterson J, et al. The Newcastle-Ottawa Scale (NOS) for assessing the quality of nonrandomised studies in meta-analyses. Accessed January 16, 2020 at: http://www.ohri.$\mathrm{ca} /$ programs/clinical_epidemiology/oxford.asp

13 Wang C, Shi YF, Xie PJ, Wu JH. Accuracy of digital complete dentures: a systematic review of in vitro studies. J Prosthet Dent 2021;125(02):249-256

14 Rosa WL, Piva E, Silva AF. Bond strength of universal adhesives: a systematic review and meta-analysis. J Dent 2015;43(07): 765-776

15 Garcia-Hammaker S, Saglik B, Sierraalta M, Razzoog M. Influence of screw channel angulation on the fracture resistance of zirconia abutments: an in vitro study. J Prosthodont 2021;30(04):329-334

16 Drew A, Randi A, DiPede L, Luke A. Fracture strength of implant screw-retained all-ceramic crowns with the use of the angulated screw channel: a pilot study. Int J Periodontics Restorative Dent 2020;40(02):245-252

17 Swamidass RS, Kan JYK, Kattadiyil MT, Goodacre CJ, Lozada J. Abutment screw torque changes with straight and angled screwaccess channels. J Prosthet Dent 2021;124(04):675-681

18 Opler R, Wadhwani C, Chung KH. The effect of screwdriver angle variation on the off-axis implant abutment system and hexalobular screw. J Prosthet Dent 2020;123(03):524-528

19 Mulla SH, Seghi RR, Johnston WM, Yilmaz B. Effect of cyclic loading on reverse torque values of angled screw channel systems. J Prosthet Dent 2021;S0022-3913(21):00007-X. Doi: 10.1016/j. prosdent.2020.12.020

20 Hu E, Petrich A, Imamura G, Hamlin C. Effect of screw channel angulation on reverse torque values of dental implant abutment screws. J Prosthodont 2019;28(09):969-972

21 Goldberg J, Lee T, Phark JH, Chee W. Removal torque and force to failure of non-axially tightened implant abutment screws. J Prosthet Dent 2019;121(02):322-326

22 Pol CWP, Raghoebar GM, Maragkou Z, Cune MS, Meijer HJA. Fullzirconia single-tooth molar implant-supported restorations with angulated screw channel abutments: a 1-year prospective case series study. Clin Implant Dent Relat Res 2020;22(01):138-144

23 Greer AC, Hoyle PJ, Vere JW, Wragg PF. Mechanical complications associated with angled screw channel restorations. Int J Prosthodont 2017;30(03):258-259

24 Friberg B, Ahmadzai M. A prospective study on single tooth reconstructions using parallel walled implants with internal connection (NobelParallel CC) and abutments with angulated screw channels (ASC). Clin Implant Dent Relat Res 2019;21(02): 226-231

25 Anitua E, Flores C, Piñas L, Alkhraisat MH. Frequency of technical complications in fixed implant prosthesis: the effect of prosthesis screw emergence correction by computer-aided design/computer-aided manufacturing. J Oral Implantol 2018; 44(06):427-431

26 Anitua E, Fernández-de-Retana S, Alkhraisat MH. Survival and marginal bone loss of dental implants supporting cad-cam angled channel restorations: a split-mouth retrospective study. Eur J Dent 2020;14(02):194-199

27 Tallarico M, Ceruso FM, Xhanari E, Gargari M, Canullo L, Meloni SM. Immediately loaded tilted implants combined with angulated screw channel zirconia abutments in atrophic maxillary patients: a three-year after loading prospective case series study. ORAL and Implantol. 2018;11:106-114

28 Shi J-Y, Lv X-L, Gu Y-X, Lai H-C. Angulated screw-retained and cemented implant crowns following flapless immediate implant placement in the aesthetic region: a 1-year prospective cohort study. Int J Oral Implantol (Berl) 2020;13(03):269-277

29 Nastri L, Nucci L, Grassia V, Miraldi R. Aesthetic outcomes and peri-implant health of angled screw retained implant restorations compared with cement retained crowns: medium term follow-up. J Funct Biomater 2021;12(02):12

30 Lin YT, Shen YF, Wei PC, Hsu KW. Clinical evaluation of two-piece zirconia abutments with bonded titanium inserts for implantsupported restorations. J Prosthet Dent 2020;123(03):449-454

31 Aboushelib MN, Salameh Z. Zirconia implant abutment fracture: clinical case reports and precautions for use. Int J Prosthodont 2009;22(06):616-619

32 Zandparsa R, Albosefi A. An in vitro comparison of fracture load of zirconia custom abutments with internal connection and different angulations and thicknesses: part II. J Prosthodont 2016;25 (02):151-155

33 Bickford JH. An Introduction to the Design and Behavior of Bolted Joints. Oxford, United Kingdom: Tylor and Francis Group; 1995: $175-180$

34 Torcato LB, Pellizzer EP, Verri FR, Falcón-Antenucci RM, Santiago Júnior JF, de Faria Almeida DA. Influence of parafunctional loading and prosthetic connection on stress distribution: a 3D finite element analysis. J Prosthet Dent 2015;114(05):644-651

35 Hurson S. Use of authentic, integrated dental implant components vital to predictability and successful long-term clinical outcomes. Compend Contin Educ Dent 2016;37(07):450-455, z456

36 Monje A, Chan HL, Suarez F, Galindo-Moreno P, Wang HL. Marginal bone loss around tilted implants in comparison to straight implants: a meta-analysis. Int J Oral Maxillofac Implants 2012;27 (06): $1576-1583$

37 Del Fabbro M, Ceresoli V. The fate of marginal bone around axial vs. tilted implants: a systematic review. Eur J Oral Implantology 2014;7(Suppl 2):S171-S189

38 Chen Z, Lin CY, Li J, Wang HL, Yu H. Influence of abutment height on peri-implant marginal bone loss: a systematic review and meta-analysis. J Prosthet Dent 2019;122(01):14-21.e2

39 Tallarico M, Caneva M, Meloni SM, Xhanari E, Covani U, Canullo L. Definitive abutments placed at implant insertion and never removed: is it an effective approach? a systematic review and meta-analysis of randomized controlled trials. J Oral Maxillofac Surg 2018;76(02):316-324

40 Anitua E, Alkhraisat MH. Clinical performance of short dental implants supporting single crown restoration in the molar-premolar region: cement versus screw retention. Int J Oral Maxillofac Implants 2019;34(04):969-976

41 Chen J-Y, Pan Y-H. Zirconia implant abutments supporting single all-ceramic crowns in anterior and premolar regions: a six-year retrospective study. Biomed J 2019;42(05):358-364

42 Guljé FL, Meijer HJA, Abrahamsson I, et al. Comparison of 6-mm and 11-mm dental implants in the posterior region supporting fixed dental prostheses: 5-year results of an open multicenter randomized controlled trial. Clin Oral Implants Res 2021;32(01): $15-22$ 
43 Hotinski E, Dudley J. Abutment screw loosening in angulationcorrecting implants: An in vitro study. J Prosthet Dent 2019;121 (01):151-155

44 Menéndez-Collar M, Serrera-Figallo MA, Hita-Iglesias $P$, et al. Straight and tilted implants for supporting screw-retained fullarch dental prostheses in atrophic maxillae: a 2-year prospective study. Med Oral Patol Oral Cir Bucal 2018;23(06):e733-e741

45 Mokhtarpour H, Eftekhar Ashtiani R, Mahshid M, Tabatabaian F, Alikhasi M. Effect of screw access hole preparation on fracture load of implant-supported zirconia-based crowns: an in vitro study. J Dent Res Dent Clin Dent Prospect 2016;10(03):181-188

46 Paolantoni G, Marenzi G, Blasi A, Mignogna J, Sammartino G. Findings of a four-year randomized controlled clinical trial comparing two-piece and one-piece zirconia abutments supporting single prosthetic restorations in maxillary anterior region. BioMed Res Int 2016;2016:8767845

47 Vélez J, Peláez J, López-Suárez C, Agustín-Panadero R, Tobar C, Suárez MJ. Influence of implant connection, abutment design and screw insertion torque on implant-abutment misfit. J Clin Med 2020;9(08):2365

48 González-Martín O, Veltri M. Immediate implant in maxillary central incisors and prosthetic screw channel: a CBCT feasibility study. Int J Periodontics Restorative Dent 2021;41(02): 245-251

49 Farré-Berga O, Cercadillo-Ibarguren I, Sánchez-Torres A, et al. Novel ball head screw and screwdriver design for implant-supported prostheses with angled channels: a finite element analysis. J Oral Implantol 2018;44(06):416-422

50 Farronato D, Manfredini M, Stevanello A, Campana V, Azzi L, Farronato M. A comparative 3D finite element computational study of three connections. Materials (Basel) 2019;12(19): 3135

51 Wang J, Lerman G, Bittner N, Fan W, Lalla E, Papapanou PN. Immediate versus delayed temporization at posterior single implant sites: A randomized controlled trial. J Clin Periodontol 2020;47(10):1281-1291 